\title{
Interactive comment on "Small-scale structure of thermodynamic phase in Arctic mixed-phase clouds observed by airborne remote sensing during a cold air outbreak and a warm air advection event” by Elena Ruiz-Donoso et al.
}

\section{Anonymous Referee \#2}

Received and published: 6 January 2020

Review of "Small-scale structure of thermodynamic phase in Arctic mixed-phase clouds observed by airborne remote sensing during a cold air outbreak and a warm air advection event" by Ruiz-Donoso et al.

Recommendation: Accept with minor revision

This paper uses a combination of active and passive remote sensing instrumentation to characterize the small scale structure of cloud thermodynamic phase using two case studies observed in the Arctic. The paper is well written, gives significant results and 
the approach seems technically sound. Thus, as such, I think it is appropriate for publication in ACP. However, there are a few minor editorial comments and some additional points that the authors might want to consider before the publication is finalized.

I would like to see more explanation on why the two particular case studies were chosen and how representative these case studies are of conditions encountered in the Arctic in general. Although cases of single-layer mixed-phase clouds do occur in the Arctic as the authors state, and although they are nice to examine from a processoriented perspective because it involves the complications of interactions between different cloud layers, past studies have suggested that multi-layer clouds and even multilayer mixed-phase clouds may be more common than these single-layer clouds. Thus, some explanation of how the results from these special cases are applicable in general to remote sensing (especially cases when active remote sensing data are not available) would be appropriate.

Were there any Doppler radar data available? Some past studies have shown that the presence of cloud top generating cells frequently occur in the Arctic (as well as in other regions) and could be responsible for some of the horizontal inhomogeneity. If such data are available, perhaps more could be said about the scales of mixing of the phases and their horizontal distributions (and the processes). This would also give more information about the resolution required for analysis.

My other concern relates to the use of hexagonal columns to characterize the ice crystals. A lot of previous studies have suggested that the majority of ice crystals in Arctic clouds, including those in mixed-phase conditions, are very irregular and not well characterized by pristine shapes. Can a more realistic assumption about the ice crystal shapes be used? Or, alternatively, there should be more discussion made about the quantitative uncertainties induced by this simplistic assumption.

Printer-friendly version

Page 4, line 30: It is not true in general that the radar reflectivity for ice is proportional to the sixth power of the ice particle size. For example, Hogan and collaborators 
have developed much better quantitative models for converting ice crystal particle size distributions to radar reflectivity.

Page 5, line 8: How can the standard profile be used in combination with the dropsonde data? Wouldn't one or the other have to be used to give the vertical profile?

Page 6, line 3: What are unrealistic values of LWP? This should be more quantitative.

Page 6, line 6: What quantitative criteria were used to identify the presence of cold air outbreaks?

Page 7, line 6: Why couldn't it also be attributed to a reduced concentration of particles rather than just small particles?

Interactive comment on Atmos. Chem. Phys. Discuss., https://doi.org/10.5194/acp-2019-960, 2019. 